\title{
From Building Corpora for Recognizing Faceted Entailment to Recognizing Relational Entailment
}

\author{
Martin Víta \\ NLP Centre, Faculty of Informatics \\ Masaryk University \\ Botanická 68a, 60200 Brno, Czech Republic \\ Email: info@martinvita.eu
}

\begin{abstract}
Recognizing textual entailment (RTE) became a well established and widely studied task. Partial textual entailmentand faceted textual entailment in particular-belong to tasks that are derived from RTE. Although there exist many annotated corpora for the original RTE problem, faceted textual entailment is in the sense of easy-accessible corpora highly neglected. In this paper, we present a semi-automatic approach to deriving corpora for faceted entailment task from a general RTE corpus using open information extraction (open IE) tools.

As a generalization of this approach and general principles of open IE, we introduce a notion of relational entailment and provide its basic properties and relations to other entailmentlike issues. We would like to introduce the problem of relational entailment as an important task with potentially wide range of real-world applications.
\end{abstract}

\section{INTRODUCTION}

$\mathbf{T}$ EXTUAL entailment is a relation is between two texts or text snippets that expresses whether one text can be inferred from the other. Textual entailment has quickly become one of the most prominent tasks of NLP with a wide spectrum of (potential) applications - including multi-document summarization, plagiarism detection, machine translation evaluation etc. In the last years, a huge effort was made in the field of approaches and algorithms for recognizing textual entailment as well as in building annotated corpora for this purpose.

Nevertheless, "classical" recognizing textual entailment is not able to treat situations when the text is only "almost entailed". A notion of partial entailment was introduced by Nielsen et al. [1] to address this problem. The original motivation arises from automatic assessment of students' tests where it is necessary to cover situations when a certain answer is only partially correct. Facets, i. e., pairs of words accompanied by a semantic relationship connecting these two words together, were introduced in the same paper in order to present a model of decomposing the sentence into fragments and provide a basis for recognizing partial entailment. Later, in SemEval 2013 challenge, a pilot task of partial - faceted entailment in particular - was introduced [2].

Recognizing textual entailment is a classification task. Supervised methods are naturally a predominant approach in this setting. Especially deep learning approaches (often ensemble methods) currently highly outperform other methods [3], [4], [5]. Nevertheless, other methods than DNN are also investigated, see [6].
Deep learning methods generally require generally a great amount of training data. For textual entailment relevant sources, i. e., annotated corpora are already available, but, in contrast, in partial/faceted entailment, researchers are facing a problem of lack of the training data. One of the main goals of this paper is to suggest a method for deriving a new corpus for faceted entailment task using open information extraction tools from an existing RTE corpus.

Based on the idea of using open information extractors, we propose a novel task of relational entailment that can be viewed as a form of a partial entailment and as a modified generalization of faceted entailment.

\section{Textual Entailment, Faceted Entailment And CORRESPONDING CORPORA}

For completeness, we briefly recall the notion of textual entailment and provide a selection of notable corpora for this task.

\section{A. Recognizing Textual Entailment - Task Definition}

Textual entailment is defined as a directional relationship between pairs of text expressions, denoted by $T$ - the entailing "Text", and $H$ - the entailed "Hypothesis". We say that $T$ entails $H$ if humans reading $T$ would typically infer that $H$ is most likely true, see [7]. Deep insight into the nature of textual entailment is provided by a current work of Korman et al. [8].

Recognizing textual entailment (RTE) is fundamentally a binary classification task to decide whether a given text $T$ entails a given hypothesis $H$ - with a "boolean" answer. Later, a modification of this, so called two-way task, appeared: no entailment category was split into two classes: unknown, where the truth of $H$ cannot be determined on the basis of $T$, and contradiction, where $T$ contradicts $H$, [9].

Example. If the text $T$ has a form of a sentence Apple says the new Intel dual-core chips improve performance by up to 39 percent over the previous single-core variety. and the hypothesis $H$ is a sentence Dual-core chips make the performance better., then a correct RTE system should label this item as ENTAILMENT. 
As already mentioned, current deep ensemble architectures are beating other architectures in RTE task. ${ }^{1}$

\section{B. Recognizing Textual Entailment - Notable Corpora}

During the last decade, several annotated corpora for RTE task were created. Among most important corpora belong:

- The Boeing-Princeton-ISI (BPI) Textual Entailment Test Suite - one of the smallest and oldest corpora that contains only 250 pairs of pairs. The value of this corpus is that it provides items "syntactically simpler but semantically challenging, with the intension of focusing more on the knowledge rather than just linguistic requirements."

- RTE-1-RTE-8 - a set of corpora used in textual challenges organized under several frameworks, starting in 2004 as PASCAL Recognizing Textual Entailment (RTE) Challenges, later as the tracks of the Text Analysis Conference (TAC) and finally as a part of SemEval challenges. First RTE-1-RTE-5 were designated as collections of standalone $(T, H)$ pairs, whereas later RTE-6RTE-8 datasets were created in order to tackle more realistic scenarios, including knowledge base population, slot filling task etc. The last one was motivated by educational purposes: to assess the correctness of a student answers. Some of RTE-x corpora were annotated in a three-way manner. A comprehensive description of these corpora is available in [9].

- The Stanford Natural Language Inference (SNLI) Corpus - a collection of 570k human-written English sentence pairs manually labeled for balanced classification with the labels entailment, contradiction, and neutral. One of the most important and the biggest corpora for RTE purpose [10].

Annotated corpora are available mostly for English, nevertheless there exist corpora for other languages such as Portuguese [11]. General issues of RTE corpora preparation, i. e., generating entailment pairs as well as connection between knowledge acquisition and RTE are discussed in [12].

\section{Partial and Faceted Entailment}

An ordered pair $(T ; H)$ forms a partial textual entailment if a fragment of the hypothesis $H$ is entailed by $T$. According to this definition, the fragment of the hypothesis is no more defined. Thus, we need to answer a question how to decompose the hypothesis into fragments. The model of facets tackles this problem.

A facet is an ordered pair of key terms $\left(w_{1}, w_{2}\right)$ (that are contained in the considered hypothesis). These terms can be single words or multi-word expressions like "carbon dioxide" etc. These terms are connected together with a semantic relation. A simplified version of this approach - used in RTE-8 - deals only with the pair of these terms without the semantic relation mentioned explicitly.

\footnotetext{
${ }^{1} \mathrm{~A}$ comprehensive list of DNN successful architectures is available at: https //nlp.stanford.edu/projects/snli/

${ }^{2}$ See: http://www.cs.utexas.edu/users/pclark/bpi-test-suite/
}

For example, if the hypothesis has the form of a sentence The water was evaporated, leaving the salt., one of corresponding facets is (evaporated, water), a different one (leaving, salt), etc.

1) Recognizing Faceted Entailment - Task definition: Recognizing faceted entailment is again a binary classification task having three inputs: text $T$, hypothesis $H$ and a facet $\left(w_{1}, w_{2}\right)$ of words that occur in the hypothesis $H$. The task consists of determining whether $T$ expresses, either directly or by implication, the same relationship between the facet words as in $H$, see [2]. Positive case is labeled by "expressed", other one as "unaddressed" (rather then entailment-no entailment).

2) SemEval 2013 Task 7 RTE-8 Corpus for Faceted Entailment: Nowadays, there exists only one widely accepted corpus for faceted entailment - a corpus used in SemEval 2013 Task 7, RTE-8: it is based on SciEntsBank corpus of student answers (together with corresponding questions and reference answers). The reference answers are broken down into the facets and annotated in order to support educational systems in recognizing when specific parts of a reference answer are expressed in the student answer, even if the reference answer is not entailed as a whole [1]. As mentioned in [2], "the correspondence between educational labels and entailment judgments was not perfect due to the difference in educational and textual entailment perspectives. Nevertheless, the two classes of assessment appeared to be sufficiently well correlated."

An item taken from SciEnts Bank / SemEval 2013

\section{Task 7, RTE-8}

QUESTION: You used several methods to separate and identify the substances in mock rocks. How did you separate the salt from the water?

STUDENT ANSWER: Let the water evaporate and the salt is left behind.

REFERENCE ANSWER: The water was evaporated, leaving the salt.

FACET: (evaporated, water)

In this case, the result is "Expressed" (thus the student's answer can be regarded as partially correct).

In contrast, when student answers "I don't know." the facet (evaporated, water) is obviously not expressed.

\section{OPEN INFORMATION EXTRACTION}

Open Information Extraction (open IE or OIE for short), introduced by Banko et al. [13], is a task of extracting (ordered) textual $n$-tuples containing relation phrase and its arguments from a given sentence. For example, from a following simple sentence "FedCSIS took place in Prague.", an open IE system (open information extractor) should extract a textual triple (FedCSIS, took place in, Prague). Unless traditional information extraction concept, open IE systems do not require a predefined list of relations (given by relation vocabularies or ontologies).

Following [14], an open information extractor can be formally defined as a function from a document, $d$, to a set of triples, $\{(\arg 1, \operatorname{rel}, \arg 2)\}$, where the $\operatorname{args}$ are noun 
phrases and rel is a textual fragment indicating an implicit, semantic relation between the two noun phrases. The extractor should produce one triple for every relation stated explicitly in the text, but is not required to infer implicit facts. (For example, from the sentence "US president Donald Trump, visited Singapore.", it is not required to obtain a triple (Donald Trump, be president of, US)), nevertheless, some of current open information extractors provide a limited functionality in this field.

New generation of open IE systems as OpenIE4 and higher are also able to extract even $n$-tuples corresponding with semantic $n$-ary relations, $n>3$, as well as nested relations (used for dealing with modal sentences like "Early scientists believed that earth is the center of the universe.", where extracting (Earth, is the center of, universe) is inappropriate all described in detail in [15].

\section{A. Open IE: Goal and Applications}

Applications of open IE can be generally divided into two main groups [15]: end user tasks and NLP end tasks. End user tasks include primarily building Open knowledge bases that allow simple querying, fact finding, as well as browsing through information extracted from a collection of textual documents and/or summarizing information about given entities. Open IE approaches also provide a particular kind of data compression (a source free text $\rightarrow$ a collection of textual tuples), that preserves important information (and is still in a human-readable form).

NLP end tasks include text comprehension, computing sentence similarity and (multi-)document summarization. On more general level, open IE results, i. e. textual tuples, can be effectively used for training word embeddings. The results of "open IE embeddings" at lexical similarity and analogy tasks outperforms embeddings obtained by using other types of contexts (like probably most widely used bag-of-words) [15].

\section{B. Open IE Tools}

Since 2007, when the concept of open IE was introduced together with the first open information extractor called TextRunner in [13], further systems were created. The following paragraphs summarize some notable systems for open IE task.

The following list contains a selection of leading systems for Open IE task:

- ReVerb, [16]

- ArgLearner and R2A2, [15]

- Ollie, [15]

- OpenIE4, [15] and its successor OpenIE5,

- ClauseIE, [17]

- Stanford openIE, [18].

\section{Deriving ANnotated CoRpus For ReCOGNizing FACETED ENTAILMENT TASK}

In this section we outline a method for generating faceted entailment items, i. e., triples in form ( $T, H$, a facet) accompanied by a label (expressed/unaddressed). We assume we already have an annotated corpus for RTE from an external source containing items in form $(T, H)$, label (entailment/no entailment).

\section{A. Description of the Method}

1) From each pair $(T, H)$ such that $T \rightarrow H$, generate positive instances $T \rightarrow h_{i}$,

2) If a pair $(T, H)$ is labeled in RTE corpus as neutral/unknown (if the corpus is annotated in a three-way manner), then generate a set of negative candidates $T \rightarrow h_{i}$ to be manually checked,

3) If a pair $(T, H)$ is not labeled not as entailed nor unrelated, then (manually) check for which $h_{i}, T \nrightarrow \rightarrow h_{i}$ and add this pair $T, h_{i}$ to negative instances

4) Perform postprocessing issues over $h_{i}$.

$h_{i}$ is an output of open information extraction on $H$, such that $h_{i}$ is a triple and each element of the triple does not contain more than two words/tokens (open information extractors are able to extract longer phrases as arguments as well as longer predicates, but they are irrelevant from our "faceted" point of view).

The postprocessing consists of removing stopwords and taking an arbitrary pair of each triple $h_{i}$.

Note that we assume that if the entailment $T \rightarrow H$ does not hold, then there is at least one facet such that faceted entailment according to $T, H$ does not hold. Although the proposed method includes a certain part of manual work, in case of preparing balanced corpus, half of the work (positive instances) is done automatically and, moreover, the negative instances can be recommended from the list of potential candidates (obtained in the third step) by some simple algorithm like [19].

\section{B. Example}

Let us consider an entailment pair T: The beetle can rub its elytra making noises in order to communicate. and $H$ : The elytra are used to make sounds. - in this case, the entailment holds. (This example was taken from SciEntsBank, [2], the original question was One function of the bess beetle's elytra (the hard, black wing set) is protection. What is another function of the elytra?).

From the sentence The elytra are used to make sounds. the open information extractor produced among others a triple: (The elytra; to make; sounds). After removing the stopwords the and to, and taking for example a pair (elytra, make), we have - together with $T$ and $H$ - a positive faceted entailment item.

\section{Challenge of Evaluation}

The quality of obtained corpus depends mainly on the the quality of the open IE system involved, as well the quality of the initial RTE corpus. Evaluation of open IE systems is generally difficult, often expressed only in term of precision, not recall. First steps in repeatable and large benchmarking of open IE systems were done by Stanovsky et al. in [20]. The issue of measuring quality of the corpus obtained by described method is a challenge for further investigations. 


\section{Novel Notion: Relational Entailment}

As mentioned earlier, faceted entailment deals with ordered pairs of words or multi-word expressions, i. e., with binary relations (or ternary relations, when the semantic relation is explicitly mentioned). In this position paper we suggest relaxing the condition that "the considered object is a binary relation" and use a general textual $n$-tuples instead.

\section{A. Task definition and Examples}

Given (i) a text $T$ (ii) a hypothesis $H$, both in a form of a sentence and (iii) an open IE text $n$-tuple $t_{H}$ extracted from the sentence $H$ (where $n \geq 3$ ), the first proposed task consists of determining whether $T$ expresses, either directly or by implication, the same relationship between exhibited by $t_{H}$ in the context of $H$ (i. e. we have again a binary classification problem).

In this case, the meaning of $t_{H}$ is "emphasized" by the context given by $H$. This definition corresponds with the original definition of faceted entailment in [21].

For practical purposes arising from potential applications we can go further and omit the role of the hypothesis $H$ and state the definition of a relational entailment.

Definition. Given a text $T$ and a textual $n$-tuple $t$, the task is to decide whether the meaning of $t$, i. e., the semantic relationship expressed by $t$ can be inferred from $T$. Similarly as in the previous case, we have a binary decision task of recognizing relational entailment - RRE for short. (Positive answer can be labeled as entailed - with notation $T \rightarrow t$, negative as non-entailed - with notation: $T \not t t$.)

Example. Given a sentence $T=$ "Patrick flew from Boston to Los Angeles with Delta Airlines with one stopover." and a textual quadruple $t_{1}=$ (Patrick, flew, from East Coast, with Delta Airlines) and $t_{2}=$ (Patrick, flew, from East Coast, to Los Angeles, via Atlanta).

Following the previous definition, we can (obviously) say that $T \rightarrow t_{1}$, and, in contrast, $T \nrightarrow t_{2}$.

\section{B. Potential Applications of Relational Entailment}

From the viewpoint of faceted entailment motivation, recognizing relational entailment has a potential application as a component for automatic assessment of students' tests: having open IE tuples extracted from the reference answer and taking student's answer as an input text, we can use a RRE system for judging whether student's answer is at least partially correct.

Next, probably more important application of recognizing relational entailment, arise from checking/proving facts in open knowledge bases, i. e., in knowledge bases that consist of open IE tuples. Open knowledge bases can be easily interpreted by humans and form an intermediate representation of information in the text(s), [15]. The RRE can be used for checking whether a given text provides an evidence for a given piece of information contained in a considered open knowledge base and subsequently in case of searching relevant text supporting certain fact of an open $\mathrm{KB}$.
Other possible applications may cover various aspects of multi-document summarization, computing textual similarity as well as some other common NLP tasks.

\section{Recognizing Relational Entailment, Recognizing Textual Entailment and Paraphrase Identification}

In [21], Levy et al. used recognizing faceted entailment system for recognizing ("complete") textual entailment: in the paper, they propose a following, three-stage architecture:

1) Decompose the hypothesis into facets.

2) Determine whether each facet is entailed.

3) Aggregate the individual facet results and decide on complete entailment accordingly.

This approach provided promising results, nevertheless it relies on the assumption that the result about complete entailment can be done (only) from the individual facet results. Generally, there exist textual tuples not entailed by the given text $T$, although the facets (as pairs of word expressions) covering the tuple are expressed/entailed from $T$.

This approach presented in [21] also assumes the facet decomposition is already performed.

Obviously, this approach can be modified in order to exploit the benefits of "open IE" perspective:

1) Instead of decomposing the hypothesis into the facets, we generate a set of open IE tuples (this set of tuples can be viewed as a "small" open knowledge base.

2) Instead of determining whether each facet is entailed, we decide, whether each open IE tuple is entailed with respect of the definition above.

3) Aggregating of individual "tuple" results.

This proposed approach brings two main advantages comparing to the faceted-like approach:

1) We leave the decomposition of the hypothesis to an open information extractor.

2) We also decide about entailment of facts that cannot be decomposed into a set of ordered pairs, i. e. facets (that could cause false positives).

By this architecture, we have already linked recognizing relational entailment with recognizing "complete" textual entailment. Now, we are able to do one more natural step towards widely investigated NLP problem: paraphrase identification. Since paraphrase identification can be viewed as a bidirectional entailment, the modification for our setting is straightforward. Let us consider we have a couple of sentences $P$ and $S$ and corresponding sets of open IE tuples $\left\{p_{1}, p_{2}, \ldots p_{n}\right\}$ and $\left\{s_{1}, s_{2}, \ldots s_{m}\right\}$, respectively. The final judgment about paraphrasing can be viewed as an aggregation of results about individual relational entailments $P \rightarrow s_{i}$ and $S \rightarrow p_{j}$, where $1 \leq i \leq n$ and $1 \leq j \leq m$.

\section{Creating Annotated Corpora for Recognizing Relational Entailment}

Similarly as by "classical" recognizing textual entailment problems, we can expect that in recognizing relational entailment challenges supervised approaches will play a key role. 
Hence there arises a need of annotated corpora for recognizing relational entailment.

Analogously as in the "facet" case, we can use corpora for recognizing textual entailment that were already built. We assume an open IE extractor is available. In contrast to the faceted case, the situation is simpler since there is no need to modify extracted textual tuples.

We can summarize the semi-automatic method of creating RRE corpus in the following steps:

1) From each pair $(T, H)$ where entailment $T \rightarrow H$ holds, generate positive instances $T \rightarrow h_{i}$,

2) If a pair $(T, H)$ is labeled in RTE corpus as neutral/unknown (if the corpus is annotated in a three-way manner), then generate a set of negative candidates $T \rightarrow h_{i}$ to be manually checked,

3) If a pair $(T, H)$ is not labeled not as entailed nor unrelated, then (manually) check for which tuple $t_{h}$ extracted from $H, T \nrightarrow \rightarrow t_{h}$ and add this pair $\left(T, t_{h}\right)$ as a negative instance.

( $h_{i}$ is an output of open information extraction on $H$ ).

Similarly as in the "facet case", we assume that if the entailment $T \rightarrow H$ does not hold, then there must be at least one textual open IE tuple such that relational entailment from $T$ does not hold. Again, half of the work in building balanced annotated corpora (positive instances) is done automatically and, moreover, the negative instances can be recommended from the list of potential candidates by some simple algorithm.

In some cases, it may be reasonable to remove pairs $\left(T, h_{i}\right)$ where the ratio "number of tokens in $h_{i} /$ number of tokens in $H$ " is higher than a given threshold in order to avoid cases where obtained tuples are mostly, in fact, permutations of tokens of the hypothesis, such as H: Slovakia is a member of $E U$. and $h_{i}$ : (Slovakia; is a member of; EU). In these cases, the task of RRE would be highly similar to "classical RTE".

Example. Let us consider a text T: A Filipino hostage in Iraq was released. and a hypothesis $H_{1}$ : The hostage is a citizen of the Philippines. labeled as entailment. An open information extractor provides a triple (The hostage; is a citizen of; the Philippines), hence we have a positive instance of relational entailment: A Filipino hostage in Iraq was released. $\rightarrow$ (The hostage; is a citizen of; the Philippines). In contrast, if the hypothesis is The hostage was a citizen of Iraq. and related extraction is (The hostage; was a citizen of; Iraq), than we got a non-entailment item for RRE corpus.

\section{CONCLUSION}

In this paper we have proposed a method for semi-automatic building of a corpus for recognizing faceted entailment when annotated corpus for RTE task is given and open information extractor is available. Current absence of mid- or large scale annotated corpora for faceted entailment is a serious restriction for using modern deep learning approaches.

Subsequently, we have established a connection between "the world of entailment" and "the world of open IE" by introducing the notion of relational entailment. We have demonstrated its strengths, possible applications and we have presented it as a natural generalization of faceted entailment.

\section{A. Discussion}

Obviously, the success of most of presented issues hardly relies on the quality of used open information extractor(s). Nowadays, evaluation and benchmarking of open IE tools become emerging and important topic [20]. In current investigations, Groth et al. note [22] that OIE systems perform significantly worse on scientific text than encyclopedic text. Thus various aspects - the domain of the text for instance should be taken into the account, and the results should be interpreted in such context.

Regarding the newly proposed notion of relational entailment, there arises a need for a sound methodology for creating annotated corpora in order to ensure a suitable quality of training data. There will be also a demand for benchmarks of potential RRE engines and employment of modern metalearning/algorithm selection issues [23].

It also should be take into the account, that proposed notions and architectures are "high level", hence the overall process of training recognizing relational entailment will be prone to error propagation from the initial levels: the quality of data used for training/tuning/development of open information extractor affects the coverage and correctness of extracted textual tuples. The quality of corpus for RRE is also influenced by the quality of RTE annotated corpus we are extracting the tuples from. The overall amount of training instances of RRE corpus then affects the quality of RRE system, that is subsequently propagated in downstream application. Thus each component of this process requires careful error analysis.

\section{B. Further Work}

Since we have proposed a new decision problem, the first part of further work will include building and improving corpora for relational entailment, create first architecture for RRE system and provide its evaluations, not only for English but also for languages where RTE corpora and open IE language models are available.

A general issue will be investigations of open IE tuples embeddings, i. e. their vector representations.

Other direction of research is building suitable corpora for faceted entailment and assessment of their quality.

\section{REFERENCES}

[1] R. D. Nielsen, W. Ward, and J. H. Martin, "Recognizing entailment in intelligent tutoring systems," Natural Language Engineering, vol. 15, no. 4, pp. 479-501, 2009. doi: 10.1017/S135132490999012X

[2] M. Dzikovska, R. D. Nielsen, C. Brew, C. Leacock, D. Giampiccolo, L. Bentivogli, P. Clark, I. Dagan, and H. T. Dang, "Semeval-2013 task 7: The joint student response analysis and 8th recognizing textual entailment challenge," NORTH TEXAS STATE UNIV DENTON, Tech. Rep., 2013.

[3] R. Ghaeini, S. A. Hasan, V. Datla, J. Liu, K. Lee, A. Qadir, Y. Ling, A. Prakash, X. Z. Fern, and O. Farri, "Dr-bilstm: Dependent reading bidirectional lstm for natural language inference," arXiv preprint arXiv:1802.05577, 2018.

[4] M. E. Peters, M. Neumann, M. Iyyer, M. Gardner, C. Clark, K. Lee, and L. Zettlemoyer, "Deep contextualized word representations," arXiv preprint arXiv:1802.05365, 2018. 
[5] Y. Tay, L. A. Tuan, and S. C. Hui, "A compare-propagate architecture with alignment factorization for natural language inference," arXiv preprint arXiv:1801.00102, 2017.

[6] R. Zanoli and S. Colombo, "A transformation-driven approach for recognizing textual entailment," Natural Language Engineering, vol. 23, no. 4, pp. 507-534, 2017. doi: 10.1017/S1351324916000176

[7] I. Dagan, O. Glickman, and B. Magnini, "The pascal recognising textual entailment challenge," in Machine learning challenges. evaluating predictive uncertainty, visual object classification, and recognising tectual entailment. Springer, 2006, pp. 177-190.

[8] D. Z. Korman, E. Mack, J. Jett, and A. H. Renear, "Defining textual entailment," Journal of the Association for Information Science and Technology, vol. 69, no. 6, pp. 763-772, 2018. doi: 10.1002/asi.24007

[9] L. Bentivogli, I. Dagan, and B. Magnini, "The recognizing textual entailment challenges: Datasets and methodologies," in Handbook of Linguistic Annotation. Springer, 2017, pp. 1119-1147.

[10] S. R. Bowman, G. Angeli, C. Potts, and C. D. Manning, "A large annotated corpus for learning natural language inference," in Proceedings of the 2015 Conference on Empirical Methods in Natural Language Processing (EMNLP). Association for Computational Linguistics, 2015.

[11] G. Rocha and H. Lopes Cardoso, "Recognizing textual entailment: Challenges in the portuguese language," Information, vol. 9, no. 4, p. 76, 2018. doi: 10.3390/info9040076

[12] M.-S. Guo, Y. Zhang, and T. Liu, "Research advances and prospect of recognizing textual entailment and knowledge acquisition," Jisuanji Xuebao/Chinese Journal of Computers, vol. 40, pp. 889-910, 042017. doi: 10.11897/SP.J.1016.2017.00889

[13] M. Banko, M. J. Cafarella, S. Soderland, M. Broadhead, and O. Etzioni, "Open information extraction from the web." in IJCAI, vol. 7, 2007, pp. 2670-2676.

[14] F. Wu and D. S. Weld, "Open information extraction using wikipedia," in Proceedings of the 48th Annual Meeting of the Association for Computational Linguistics. Association for Computational Linguistics, 2010, pp. 118-127.
[15] M. Mausam, "Open information extraction systems and downstream applications," in Proceedings of the Twenty-Fifth International Joint Conference on Artificial Intelligence. AAAI Press, 2016, pp. 40744077.

[16] A. Fader, S. Soderland, and O. Etzioni, "Identifying relations for open information extraction," in Proceedings of the conference on empirical methods in natural language processing. Association for Computational Linguistics, 2011, pp. 1535-1545.

[17] L. Del Corro and R. Gemulla, "Clausie: clause-based open information extraction," in Proceedings of the 22nd international conference on World Wide Web. ACM, 2013, pp. 355-366.

[18] G. Angeli, M. J. J. Premkumar, and C. D. Manning, "Leveraging linguistic structure for open domain information extraction," in Proceedings of the 53rd Annual Meeting of the Association for Computational Linguistics and the 7th International Joint Conference on Natural Language Processing (Volume 1: Long Papers), vol. 1, 2015, pp. 344354.

[19] M. Víta and V. Kríž, "Word2vec based system for recognizing partial textual entailment," in Computer Science and Information Systems (FedCSIS), 2016 Federated Conference on. IEEE, 2016, pp. 513-516.

[20] G. Stanovsky and I. Dagan, "Creating a large benchmark for open information extraction," in Proceedings of the 2016 Conference on Empirical Methods in Natural Language Processing, 2016, pp. 23002305.

[21] O. Levy, T. Zesch, I. Dagan, and I. Gurevych, "Recognizing partial textual entailment," in Proceedings of the 51st Annual Meeting of the Association for Computational Linguistics (Volume 2: Short Papers), vol. 2, 2013, pp. 451-455.

[22] P. Groth, M. Lauruhn, A. Scerri, and R. Daniel Jr, "Open information extraction on scientific text: An evaluation," arXiv preprint arXiv:1802.05574, 2018.

[23] P. Brazdil and C. Giraud-Carrier, "Metalearning and algorithm selection: progress, state of the art and introduction to the 2018 special issue," 2018. 